\title{
GEOMETRICAL CHARACTERISTICS OF GAUSSIAN SEA WAVES
}

\author{
JEAN-MARC AZAÏS, ${ }^{*}$ Université Paul Sabatier \\ JOSÉ R. LEÓN, ${ }^{* * * * *}$ Universidad Central de Venezuela \\ JOAQUÍN ORTEGA, ${ }^{* * * * * *}$ CIMAT and Universidad Central de Venezuela
}

\begin{abstract}
In this work, we study some geometrical properties of a stationary Gaussian field modeling the sea surface, using the energy spectrum. We consider the length of a crest and the mean speed of contours, which can be expressed as integrals over level sets. We also give central limit theorems for some of these quantities, using chaos expansions.
\end{abstract}

Keywords: Gaussian process; velocity level curve; crest; central limit theorem

2000 Mathematics Subject Classification: Primary 60G15

Secondary $60 \mathrm{~K} 40$

\section{Introduction}

In this work, we study some geometrical properties of the random surface represented by the level of the sea. We model the sea level using a Gaussian process and deduce its properties from the energy spectrum, which can be recorded by a measurement device. The main quantities we are interested in are

- the length of a crest, i.e. a set of local maxima in a given direction, and

- the mean speed of contours.

These quantities can be expressed as integrals over level sets.

In Section 2, we describe the usual model of the level set. In Section 3, we introduce a representation of the integral over level sets and establish Rice's formulae for its expectation. Sections 4 and 5 are devoted to the study of the crest and the speed of contours, respectively. In Section 6, we establish central limit theorems for these quantities, using chaos expansions. All of our results are illustrated in Section 7, using actual spectrum data.

Our main source of inspiration is the seminal 1957 paper 'The statistical analysis of a random moving surface' by M. S. Longuet-Higgins, in which the sea surface was modeled for the first time as a stationary Gaussian field. The Rice formulae that we obtain were deduced from heuristic considerations by Longuet-Higgins. Our work owes much to his ideas. Our second source of inspiration is the work done in the 1980s by Benzaquen and Cabaña (1982), Cabaña (1985), (1987), and Wschebor (1982), (1985). These authors established, in a slightly different form, several formulae that we reconsider in this paper.

Received 26 February 2003; revision received 19 October 2004.

* Postal address: Laboratoire de Statistique et Probabilités, Université Paul Sabatier, 118, Route de Narbonne, 31062 Toulouse cedex, France. Email address: azais@cict.fr

** Postal address: Facultad de Ciencias, Universidad Central de Venezuela, Caracas 1020, Venezuela.

*** Email address: jleon@euler.ciens.ucv.ve

**** Email address: jortega@ cimat.mx 
Let us now establish some notation. By $p_{X}(\boldsymbol{x})$ we denote the density of the random vector $\boldsymbol{X}$ at $\boldsymbol{x}$, when it exists; by $|Q|$ we denote the Lebesgue measure of the set $Q$; and by $\mathbf{1}_{E}$ we denote the indicator function of the set $E$.

\section{Modeling the sea surface}

Neglecting general factors like tides and surge, the height of the sea with respect to a reference level can be modeled by a stationary Gaussian process. This level depends on two space variables $(x, y)$ and a time variable $t$, and will be denoted $\zeta(x, y, t)$. Since deterministic components have been removed, this process can be supposed to have zero mean.

Stationary Gaussian processes can be viewed as an infinite sum of elementary waves of the form

$$
\zeta_{n}(x, y, t)=\operatorname{Re}\left(C_{n} \mathrm{e}^{\mathrm{i}\left(\lambda_{1, n} x+\lambda_{2, n} y+\omega_{n} t\right)}\right),
$$

where $C_{n}$ is a complex normal variable, $\lambda_{1, n}, \lambda_{2, n}$, and $\omega_{n}$ are the parameters of some spatio-temporal oscillation, and Re denotes the real part. Assuming infinite depth, this wave must satisfy the Airy relation (see Ochi (1998) or Krée and Soize (1983))

$$
\frac{\omega_{n}^{2}}{g}=\sqrt{\lambda_{1, n}^{2}+\lambda_{2, n}^{2}}
$$

where $g$ is the acceleration due to gravity, which we choose, without loss of generality, to be $g=1$. Taking the integral limit of an infinite sum of terms of the form (1), and assuming that the spectral density exists, we obtain

$$
\zeta(x, y, t)=\int_{\Lambda} \mathrm{e}^{\mathrm{i}\left(\lambda_{1} x+\lambda_{2} y+\omega t\right)} \sqrt{f\left(\lambda_{1}, \lambda_{2}\right)} \mathrm{d} W\left(\lambda_{1}, \lambda_{2}\right),
$$

where $\Lambda$ is the manifold $\left\{\lambda_{1}^{2}+\lambda_{2}^{2}=\omega^{4}\right\}, f$ is symmetric with respect to the origin and $\mathrm{d} W$ is a complex white noise that satisfies $\mathrm{d} W\left(-\lambda_{1},-\lambda_{2}\right)=\mathrm{d} W\left(\lambda_{1}, \lambda_{2}\right)^{*}$. The covariance function of this process is given by

$$
r(x, y, t)=\int_{\Lambda} \mathrm{e}^{\mathrm{i}\left(\lambda_{1} x+\lambda_{2} y+\omega t\right)} f\left(\lambda_{1}, \lambda_{2}\right) \mathrm{d} \lambda_{1} \mathrm{~d} \lambda_{2} .
$$

Making the changes of variable $\lambda_{1}=\omega^{2} \cos \varphi$ and $\lambda_{2}=\omega^{2} \sin \varphi$, we obtain the following representation, in terms of the directional spectral density $G$ (again, see Ochi (1998) or Krée and Soize (1983)):

$$
r(x, y, t)=\int_{-\infty}^{\infty} \int_{0}^{2 \pi} \exp \left\{\mathrm{i}\left(\omega^{2}(x \cos \varphi+y \sin \varphi)+\omega t\right)\right\} G(\varphi, \omega) \mathrm{d} \varphi \mathrm{d} \omega .
$$

\section{Representation of level integrals}

In this section, $Z(x, y)$ is a centered, stationary Gaussian process with variance 1 and $C^{3}$ paths and spectral density, with representation

$$
Z(x, y)=\int_{\mathbb{R}^{2}} \mathrm{e}^{\mathrm{i}\left(\lambda_{1} x+\lambda_{2} y\right)} \sqrt{f\left(\lambda_{1}, \lambda_{2}\right)} \mathrm{d} W\left(\lambda_{1}, \lambda_{2}\right) .
$$


This representation can be obtained from (2) by fixing the time. We define the spectral moments as

$$
m_{p q}=\int_{\mathbb{R}^{2}} \lambda_{1}^{p} \lambda_{2}^{q} f\left(\lambda_{1}, \lambda_{2}\right) \mathrm{d} \lambda_{1} \mathrm{~d} \lambda_{2}
$$

These moments exist at least for $p+q \leq 6$, since the process has $C^{3}$ paths.

With $u$ a level and $Q$ a set of values of $(x, y)$ with zero boundary measure, we define the level set

$$
C_{Q}(u)=\{(x, y) \in Q: Z(x, y)=u\} .
$$

The local inverse theorem implies that this set is a regular curve and that the normal $\boldsymbol{n}$ to this set (taken in the direction of increasing $Z$ ) is well defined. We will prove the following facts.

Assertion 1. Let $\boldsymbol{v}(x, y)=\left(v_{1}(x, y), v_{2}(x, y)\right)$ be a bounded vector field with a set of discontinuities of measure 0 , and let $g$ be a continuous bounded function. Then,

$$
\int_{\mathbb{R}} g(u) \mathrm{d} u \int_{C_{Q}(u)}\langle\boldsymbol{v}, \boldsymbol{n}\rangle \mathrm{d} \sigma=\int_{Q} g(Z(x, y))\langle\boldsymbol{v}(x, y), \nabla Z(x, y)\rangle \mathrm{d} x \mathrm{~d} y \text { almost surely, }
$$

where $\mathrm{d} \sigma$ is the curve length differential.

We write $\nabla Z(x, y)=\|\nabla Z(x, y)\|(\cos \varphi(x, y), \sin \varphi(x, y))$ and omit the argument $(x, y)$ for ease of notation. Consider the case in which

$$
\boldsymbol{v}=\gamma(\varphi) \frac{\nabla Z}{\|\nabla Z\|},
$$

for some function $\gamma$. Adler (1981) has shown that the zeros of $\|\nabla Z\|$ are isolated, so that $v$ verifies the condition needed for formula (3). Thus, this formula becomes

$$
\int_{\mathbb{R}} g(u) \mathrm{d} u \int_{C_{Q}(u)} \gamma(\varphi) \mathrm{d} \sigma=\int_{Q} g(Z) \gamma(\varphi)\|\nabla Z\| \mathrm{d} x \mathrm{~d} y \text { almost surely. }
$$

Let $\mathcal{L}\left(C_{Q}(u)\right)$ be the length of the level set $C_{Q}(u)$. If $\gamma(\varphi) \equiv 1$ in (5), we obtain the following formula, which can be found in Federer (1969):

$$
\int_{\mathbb{R}} g(u) \mathcal{L}\left(C_{Q}(u)\right) \mathrm{d} u=\int_{Q} g(Z)\|\nabla Z\| \mathrm{d} x \mathrm{~d} y \text { almost surely. }
$$

Assertion 2. Taking expectations on both sides of (5), we get the Rice formula for a vector field $v$ of the form (4), i.e.

$$
\begin{aligned}
& \mathrm{E}\left[\int_{C_{Q}(u)} \gamma(\varphi) \mathrm{d} \sigma\right] \\
& \quad=|Q| p(u) \mathrm{E}[\|\nabla Z(0,0)\| \gamma(\varphi(0,0))] \\
& \quad=|Q| p(u) \iint_{\mathbb{R}^{2}} \frac{\sqrt{x^{2}+y^{2}}}{2 \pi|\Sigma|^{1 / 2}} \gamma\left(\arctan \frac{y}{x}\right) \exp \left\{-\frac{1}{2}(x, y) \Sigma^{-1}(x, y)^{\top}\right\} \mathrm{d} x \mathrm{~d} y,
\end{aligned}
$$

where $p(u)$ stands for the density of $Z(x, y)$ at any point $(x, y), \Sigma$ is the covariance matrix of the Gaussian vector $\nabla Z(0,0)$, and $|\Sigma|$ its determinant. By diagonalizing $\Sigma^{-1}$ and going to polar coordinates, we find that this expression is equal to

$$
|Q| \frac{1}{2 \sqrt{2 \pi}} p(u)|\Sigma| \int_{0}^{2 \pi} \gamma(\varphi)(l(\varphi))^{-3 / 2} \mathrm{~d} \varphi
$$

where $l(\varphi)=m_{20} \cos ^{2} \varphi-2 m_{11} \cos \varphi \sin \varphi+m_{02} \sin ^{2} \varphi$. 
When $\gamma(\varphi) \equiv 1$, we obtain

$$
\mathrm{E}\left[\mathcal{L}\left(C_{Q}(u)\right)\right]=|Q| p(u) \mathrm{E}\|\nabla Z(0,0)\|=\sqrt{\frac{2}{\pi}}|Q| p(u) \sqrt{\gamma_{2}} \&(k),
$$

where $\gamma_{1}$ and $\gamma_{2}, \gamma_{2}>\gamma_{1}$, are the eigenvalues of $\Sigma, k^{2}=\left(1-\gamma_{1} / \gamma_{2}\right)$ and $\mathcal{E}(k)=$ $\int_{0}^{\pi / 2}\left(1-k^{2} \sin ^{2} \varphi\right)^{1 / 2} \mathrm{~d} \varphi$ is the elliptic integral of the first kind. Longuet-Higgins (1957, Equation (2.3.13)) showed this result for the first time, using a different notation. His version is

$$
\mathrm{E}\left[\mathcal{L}\left(C_{Q}(u)\right)\right]=\sqrt{\frac{2}{\pi}} p(u)\left(m_{20}+m_{02}\right)^{1 / 2}\left(1+\left(\frac{m_{02}}{m_{20}}\right)\right)^{-1 / 2} \mathcal{E}\left(\sqrt{1-\left(\frac{m_{02}}{m_{20}}\right)}\right),
$$

where he has supposed that $|Q|=1$ and $m_{11}=0$, in which case $\gamma_{2}=m_{20}$ and $\gamma_{1}=m_{02}$.

Remark 1. Formula (8) gives a generalization of Corrsin's formula (Corrsin (1955)) to every level $u$. This formula was established for $u=0$ in a different manner. It says that

$$
\frac{\mathrm{E}\left[\mathcal{L}\left(C_{Q}(u)\right)\right]}{|Q|}=\frac{1}{4} \int_{0}^{2 \pi} \tilde{\mathrm{E}}\left(N_{u}^{\theta}\right) \mathrm{d} \theta
$$

where $\tilde{\mathrm{E}}\left(N_{u}^{\theta}\right)$ is the expectation per unit of space of the number of crossings in the direction $\theta$. By Rice's formula (Cramér and Leadbetter (1967)),

$$
\tilde{\mathrm{E}}\left(N_{u}^{\theta}\right)=\sqrt{\frac{2 m_{2, \theta}}{\pi}} p(u),
$$

where $m_{2, \theta}$ is the second spectral moment in the direction $\theta$. Without loss of generality, we can assume that the direction in the plane has been chosen to diagonalize the covariance matrix of $\nabla Z$. Then,

$$
\boldsymbol{\Sigma}(\theta)=\left(\begin{array}{cc}
\gamma_{2} & 0 \\
0 & \gamma_{1}
\end{array}\right)
$$

and $m_{2, \theta}=\left[\gamma_{2}\left(1-\left(1-\gamma_{1} / \gamma_{2}\right) \sin ^{2} \theta\right)\right]^{1 / 2}$, so that the left-hand term in (8) is equal to

$$
p(u) \sqrt{\frac{2}{\pi}} \sqrt{\gamma_{2}} \int_{0}^{\pi / 2}\left(1-\left(1-\frac{\gamma_{1}}{\gamma_{2}}\right) \sin ^{2} \theta\right)^{1 / 2} \mathrm{~d} \theta=p(u) \sqrt{\frac{2}{\pi}} \sqrt{\gamma_{2}} \&(k) .
$$

Proof of Assertion 1. For now, we suppose that $Q=[0, M] \times[0, M]$ and that $v$ is differentiable and vanishes on the boundary of $Q$. Since the process $Z$ has continuous paths and variance bounded from below, by Ylvisaker's theorem (Ylvisaker (1968)), there are no local maxima at level $u$. This can be shown (see, for example, Wschebor $(1985$, p. 28)) to imply that the boundary of the set $\{(x, y) \in Q: Z(x, y) \leq u\}$ is $C_{Q}(u)$. Using Green's theorem,

$$
\begin{aligned}
\int_{\mathbb{R}} g(u) \mathrm{d} u \int_{C_{Q}(u)}\langle\boldsymbol{v}, \boldsymbol{n}\rangle \mathrm{d} \sigma & =\int_{\mathbb{R}} g(u) \mathrm{d} u \int_{\{(x, y) \in Q: Z(x, y) \leq u\}} \operatorname{div} \boldsymbol{v} \mathrm{d} x \mathrm{~d} y \\
& =\int_{Q}\left[\int_{Z(x, y)}^{\infty} g(u) \mathrm{d} u\right]\left(\frac{\partial v_{1}}{\partial x}+\frac{\partial v_{2}}{\partial y}\right) \mathrm{d} x \mathrm{~d} y=: A .
\end{aligned}
$$


Let us consider the contribution of $\partial v_{1} / \partial x$ to the integral in $x$. Integrating by parts, it is

$$
\int_{0}^{M} g(Z(x, y)) \frac{\partial Z}{\partial x} v_{1} \mathrm{~d} x
$$

so that

$$
A=\int_{0}^{M} \int_{0}^{M} g(Z(x, y))\left(\frac{\partial Z}{\partial x} v_{1}+\frac{\partial Z}{\partial y} v_{2}\right) \mathrm{d} x \mathrm{~d} y=\int_{Q} g(Z(x, y))\langle\boldsymbol{v}, \nabla Z\rangle \mathrm{d} x \mathrm{~d} y
$$

and relation (3) holds when $\boldsymbol{v}$ vanishes on the boundary.

If $\boldsymbol{v}$ does not vanish on the boundary then, since it is bounded, it is summable over $Q$, and there exists a sequence of differentiable functions $\boldsymbol{v}_{n}$ vanishing on the boundary such that $\boldsymbol{v}_{n} \rightarrow \boldsymbol{v}$ in $L^{1}$ and $\left\|\boldsymbol{v}_{n}(x, y)\right\| \leq k$, where $k$ is the bound for $\|\boldsymbol{v}(x, y)\|$ for all $(x, y)$ in $Q$. Thus, passing to the limit, (3) holds for general $\boldsymbol{v}$.

When $Q$ is not a square, it is possible to apply Rice's formula (Cramér and Leadbetter (1967)) to the stochastic process obtained by considering $Z(x, y)$ on a vertex $V$ of $Q$. We can see that $C_{Q}(u) \cap V$ is almost surely composed of a finite number of points and, thus, does not contribute to the integral in (3). Hence, both sides of (3) are almost surely additive in $Q$. Since $Q$ has zero boundary measure, it can be approximated from above and from below by two finite sums of squares with arbitrarily close measures. This proves (3) for general $Q$.

Proof of Assertion 2. Since $Z$ is stationary, its value and its derivative at a given point $(x, y)$ are independent. Taking expectations in (3), we have

$$
\begin{aligned}
\int_{\mathbb{R}} g(u) \mathrm{d} u \mathrm{E}\left[\int_{C_{Q}(u)}\langle\boldsymbol{v}, \boldsymbol{n}\rangle \mathrm{d} \sigma\right] & =\int_{Q} \int_{\mathbb{R}} g(u) p_{\boldsymbol{Z}(x, y)}(u) \mathrm{E}[\langle\nabla Z(x, y), \boldsymbol{v}(x, y)\rangle] \mathrm{d} x \mathrm{~d} y \mathrm{~d} u \\
& =|Q| \int_{\mathbb{R}} g(u) p(u) \mathrm{E}[\|\nabla Z(0,0)\| \gamma(\varphi(0,0))] \mathrm{d} u,
\end{aligned}
$$

using (4).

If $g$ is any function, duality implies that (6) is true for almost every $u$. This was first proved by Zähle (1984) in the case $\boldsymbol{v}=\boldsymbol{n}$. The right-hand side of (6) is clearly a continuous function of $u$, but it is not easy to show that the left-hand side is. The fact that (6) holds for every $u$ has been proved in a different way by Benzaquen and Cabaña (1982), Cabaña (1985), and Wschebor (1982), (1985), and we refer the reader to them for this case; also see Azaiis and Wschebor (2005). Longuet-Higgins (1957) proved (7) and said that this means the direction of the gradient has density equal to $(l(\varphi))^{-3 / 2}$. This remark must be reinterpreted using the ergodic theorem. Formula (8) was also considered by Longuet-Higgins and definitively proved by Benzaquen and Cabaña (1982) and Wschebor (1985), including the case in which the parameter space has more than two dimensions.

\section{Length of a crest}

A crest will be defined as a local maximum in a given direction, say $\theta$, of the sea surface modeled as in Section 2.

We first define a static crest at a fixed time (say $t=0$ ) as

$$
C_{Q, \theta}^{\mathrm{s}}=\left\{(x, y) \in Q: \zeta_{\theta}^{\prime}(x, y)=0, \zeta_{\theta \theta}^{\prime \prime}(x, y)<0\right\},
$$


where $\zeta_{\theta}^{\prime}$ and $\zeta_{\theta \theta}^{\prime \prime}$ are, respectively, the first and second derivatives of the field $\zeta$ in the $\theta$ direction of the $(x, y)$ plane at the point $(x, y, 0)$. Since $\theta$ is the direction of a straight line, it can be chosen in $[0, \pi)$.

We define the moving crest as

$$
\begin{aligned}
C_{Q, T, \theta}^{\mathrm{m}}=\{(z \cos \theta, z \sin \theta, t) & \in Q \times[0, T]: \\
& \left.\zeta_{\theta}^{\prime}(z \cos \theta, z \sin \theta, t)=0, \zeta_{\theta \theta}^{\prime \prime}(z \cos \theta, z \sin \theta, t)<0\right\} .
\end{aligned}
$$

We will prove the following relations.

Assertion 3. $\mathrm{E}\left[\mathcal{L}\left(C_{Q, \theta}^{\mathrm{s}}\right)\right]=\left[|Q|\left(\gamma_{2}\right)^{1 / 2} / 2 \pi\left(a_{11}\right)^{1 / 2}\right] \mathcal{E}(k)$, where, recall, $k=\left(1-\gamma_{1} / \gamma_{2}\right)^{1 / 2}$ and

$$
a_{11}=\mathrm{E}\left[\zeta_{\theta}^{\prime}(0,0)^{2}\right]=m_{20} \cos ^{2} \theta+2 m_{11} \cos \theta \sin \theta+m_{02} \sin ^{2} \theta .
$$

Here, $\gamma_{1}$ and $\gamma_{2}, \gamma_{2}>\gamma_{1}$, are the eigenvalues of the matrix

$$
\boldsymbol{\Sigma}(\theta)=\left(\begin{array}{ll}
a_{22} & a_{23} \\
a_{23} & a_{33}
\end{array}\right),
$$

in which

$$
\begin{aligned}
a_{22}= & m_{40} \cos ^{4} \theta+m_{04} \sin ^{4} \theta+6 m_{22} \cos ^{2} \theta \sin ^{2} \theta+4 m_{31} \cos ^{3} \theta \sin \theta+4 m_{13} \cos \theta \sin ^{3} \theta, \\
a_{33}= & \left(m_{40}+m_{04}\right) \cos ^{2} \theta \sin ^{2} \theta+m_{22}\left[\left(\cos ^{2} \theta-\sin ^{2} \theta\right)^{2}-2 \cos ^{2} \theta \sin ^{2} \theta\right] \\
& +2\left(m_{13}-m_{31}\right) \cos \theta \sin \theta\left(\cos ^{2} \theta-\sin ^{2} \theta\right), \\
a_{23}= & -m_{40} \cos ^{3} \theta \sin \theta+m_{31} \cos ^{2} \theta\left(\cos ^{2} \theta-3 \sin ^{2} \theta\right)+3 m_{22} \cos \theta \sin \theta\left(\cos ^{2} \theta-\sin ^{2} \theta\right) \\
& +m_{13} \sin ^{2} \theta\left(3 \cos ^{2} \theta-\sin ^{2} \theta\right)+m_{04} \cos \theta \sin ^{3} \theta,
\end{aligned}
$$

and $m_{p q}=\int_{-\infty}^{\infty} \int_{-\infty}^{\infty} \lambda_{1}^{p} \lambda_{2}^{q} f\left(\lambda_{1}, \lambda_{2}\right) \mathrm{d} \lambda_{1} \mathrm{~d} \lambda_{2}$. Although $\gamma_{i}, k$, and $a_{i j}$ all depend on $\theta$, we have not indicated these dependencies, for clarity of notation.

Assertion 4. $\mathrm{E}\left[\mathcal{L}\left(C_{Q, T, \theta}^{\mathrm{m}}\right)\right]=\left[\left|Q_{\theta}\right| T\left(\tilde{\gamma}_{2}\right)^{1 / 2} / 2 \pi\left(a_{11}\right)^{1 / 2}\right] \mathcal{E}(\tilde{k})$, where $\left|Q_{\theta}\right|$ is the length of the section of $Q$ in the $\theta$ direction, and $\tilde{\gamma}_{2}$ and $\tilde{k}$ are defined similarly to $\gamma_{2}$ and $k$, using the matrix

$$
\tilde{\boldsymbol{\Sigma}}(\theta)=\left(\begin{array}{ll}
a_{22} & \tilde{a}_{23} \\
\tilde{a}_{23} & \tilde{a}_{33}
\end{array}\right),
$$

in which

$$
\begin{aligned}
& \tilde{a}_{33}=m_{202} \cos ^{2} \theta+2 m_{112} \cos \theta \sin \theta+m_{022} \sin ^{2} \theta, \\
& \tilde{a}_{23}=m_{301} \cos ^{3} \theta+3 m_{211} \cos ^{2} \theta \sin \theta+3 m_{121} \cos \theta \sin ^{2} \theta+m_{031} \sin ^{3} \theta,
\end{aligned}
$$

and $m_{i j k}=\int_{-\infty}^{\infty} \int_{-\infty}^{\infty} \lambda_{1}^{i} \lambda_{2}^{j}\left(\lambda_{1}^{2}+\lambda_{2}^{2}\right)^{k / 4} f\left(\lambda_{1}, \lambda_{2}\right) \mathrm{d} \lambda_{1} \mathrm{~d} \lambda_{2}$. Note that $m_{i j}=m_{i j 0}$.

Proof of Assertion 3. Let us first prove the formula for a static crest. Suppose, for now, that $\theta=0$, denote by $S(x, y)$ the process $\zeta(x, y, 0)$, and let $Z(x, y)=\partial S / \partial x(x, y)$. Then, $C_{Q, 0}^{\mathrm{s}}$ can be written as

$$
C_{Q, 0}^{\mathrm{s}}=\left\{(x, y) \in Q: Z(x, y)=0, Z_{x}^{\prime}(x, y)<0\right\},
$$


where $Z_{x}^{\prime}(x, y)$ stands for $\partial Z / \partial x(x, y)$. Thus,

$$
\mathrm{E}\left[\mathcal{L}\left(C_{Q, 0}^{\mathrm{s}}\right)\right]=\mathrm{E} \int_{C_{Q}^{Z}(0)} \mathbf{1}_{\left\{Z_{x}^{\prime}(x, y)<0\right\}} \mathrm{d} \sigma,
$$

where $C_{Q}^{Z}(0)=\{(x, y) \in Q: Z(x, y)=0\}$. Since $Z^{\prime}$ and $-Z^{\prime}$ have the same distribution,

$$
\mathrm{E}\left[\mathcal{L}\left(C_{Q, 0}^{\mathrm{s}}\right)\right]=\mathrm{E} \int_{C_{Q}^{Z}(0)} \mathbf{1}_{\left\{Z_{x}^{\prime}(x, y) \geq 0\right\}} \mathrm{d} \sigma=\frac{1}{2} \mathrm{E}\left[\mathcal{L}\left(C_{Q}^{Z}(0)\right)\right] .
$$

By applying (8) we obtain

$$
\mathrm{E}\left[\mathcal{L}\left(C_{Q, 0}^{\mathrm{s}}\right)\right]=\frac{1}{2 \pi} \frac{|Q|}{\left(a_{11}\right)^{1 / 2}} \sqrt{\gamma_{2}} \mathcal{E}(k),
$$

where $a_{11}=\operatorname{var} Z(x, y)$ and $\gamma_{1}$ and $\gamma_{2}, \gamma_{2}>\gamma_{1}$, are the eigenvalues of the variance matrix of $\nabla Z$.

If $\theta \neq 0$ then $Z(x, y)$ is the process

$$
\frac{\partial \zeta}{\partial x}(x, y, 0) \cos \theta+\frac{\partial \zeta}{\partial y}(x, y, 0) \sin \theta .
$$

Hence,

$$
\begin{aligned}
& a_{11}=\operatorname{var} Z(x, y)=m_{20} \cos ^{2} \theta+2 m_{11} \cos \theta \sin \theta+m_{02} \sin ^{2} \theta, \\
& a_{22}=\operatorname{var} Z_{\theta}^{\prime}(x, y) \text { and } a_{33}=\operatorname{var} Z_{\hat{\theta}}^{\prime}(x, y),
\end{aligned}
$$

where

$$
\begin{aligned}
& Z_{\theta}^{\prime}(x, y)=\cos ^{2} \theta \frac{\partial^{2} \zeta}{\partial x^{2}}+2 \cos \theta \sin \theta \frac{\partial^{2} \zeta}{\partial x \partial y}+\sin ^{2} \theta \frac{\partial^{2} \zeta}{\partial y^{2}} \\
& Z_{\hat{\theta}}^{\prime}(x, y)=-\frac{\partial^{2} \zeta}{\partial x^{2}} \cos \theta \sin \theta+\frac{\partial^{2} \zeta}{\partial x \partial y}\left(\cos ^{2} \theta-\sin ^{2} \theta\right)+\frac{\partial^{2} \zeta}{\partial y^{2}} \cos \theta \sin \theta
\end{aligned}
$$

$\hat{\theta}$ is the direction orthogonal to $\theta$, and $a_{23}=\operatorname{cov}\left(Z_{\theta}^{\prime}, Z_{\hat{\theta}}^{\prime}\right)$.

Proof of Assertion 4. For the case of a moving crest, the proof is essentially the same, but with the following changes.

- The size of the parameter set is now $\left|Q_{\theta}\right| T$.

- The expressions for $a_{11}$ and $a_{22}$ do not change, but the gradient is now taken in the directions $\theta$ and $t$, i.e. using $\zeta_{\theta t}^{\prime \prime}(x, 0, t)=\cos \theta \zeta_{x t}^{\prime \prime}+\sin \theta \zeta_{y t}^{\prime \prime}$, we have

$$
\begin{aligned}
& \tilde{a}_{33}=\operatorname{var}\left(\zeta_{\theta t}^{\prime \prime}(x, y, t)\right), \\
& \tilde{a}_{23}=\operatorname{cov}\left(\zeta_{\theta \theta}^{\prime \prime}, \zeta_{\theta t}^{\prime \prime}\right),
\end{aligned}
$$

and we recover the desired expression.

Remarks 2. (i) When $\zeta$ is an elementary wave of the form (1), direct computations on the sine-cosine process (the elementary random wave with real parameters) show that

$$
\mathrm{E}\left[\mathcal{L}\left(C_{Q, 0}^{\mathrm{s}}\right)\right]=\frac{|Q| \sqrt{\lambda_{1}^{2}+\lambda_{2}^{2}}}{2 \pi} .
$$

Thus, the length of the crest is a nonlinear functional of the spectrum. 
(ii) We will call $r_{\theta}(x, y)$ the covariance of the process

$$
\frac{\partial \zeta}{\partial x}(x, y, 0) \cos \theta+\frac{\partial \zeta}{\partial y}(x, y, 0) \sin \theta .
$$

\section{Speed of crossings and contours}

In this section, we give a more rigorous basis to some heuristic considerations of LonguetHiggins (1957). Other approaches to the same problem have been proposed in Podgórski et al. (2000) and Baxevani et al. (2003), where several notions of velocity were introduced, including the one used here: 'velocity in the direction of the gradient'. Our results are different in the sense that we look at the two components of the gradient, while the cited authors expressed their results in terms of the joint distribution of the modulus and the angle.

\subsection{Speed of crossings}

Let $y$ be fixed (say $y=0$ ). We want to study the speed of crossing of a given level $u$ chosen 'at random' among all the crossings. Define $Q_{0}$ as the section of $Q$ in the direction of the $x$ axis. Using stationarity, it is always possible to suppose that $Q_{0}=[0, M]$ for some $M$. Also by stationarity, we can look at the speed of the sea at time 0 . A crossing is a point $x$ such that

$$
\zeta(x, 0,0)=u .
$$

The expectation of the number of crossings $N_{u}$ is given by Rice's formula (Cramér and Leadbetter (1967)):

$$
\mathrm{E}\left(N_{u}\right)=M \sqrt{\frac{2 m_{200}}{\pi}} p(u)=: D .
$$

The speed of such crossings can be computed using the implicit function theorem. From (9) we find that

$$
C_{x}(x):=\frac{\mathrm{d} x}{\mathrm{~d} t}=-\frac{\zeta_{t}^{\prime}(x, 0,0)}{\zeta_{x}^{\prime}(x, 0,0)}
$$

the mean number of crossings with speed $C_{x}$ in the interval $\left[\alpha_{1}, \alpha_{2}\right]$ can also be computed, using a Rice formula, to be

$$
\mathrm{E}\left(N_{u} \mathbf{1}_{\left\{C_{x} \in\left[\alpha_{1}, \alpha_{2}\right]\right\}}\right)=\int_{\alpha_{1}}^{\alpha_{2}} \mathrm{~d} c \int_{0}^{M} \mathrm{~d} x \int_{-\infty}^{\infty}\left|x^{\prime}\right| p_{\zeta, \zeta_{x}^{\prime}, C_{x}}\left(u, x^{\prime}, c\right) \mathrm{d} x^{\prime}=: N,
$$

where $p_{\zeta, \zeta_{x}^{\prime}, C_{x}}$ is the joint density of $\left(\zeta(x, 0,0), \zeta_{x}^{\prime}(x, 0,0), C_{x}(x)\right)$ and, by stationarity, does not depend on $x$. For the same reason, the values of the process and its derivatives at a given point are independent. Thus,

$$
N=\operatorname{Mp}(u) \int_{\alpha_{1}}^{\alpha_{2}} \mathrm{~d} c \int_{-\infty}^{\infty}\left|x^{\prime}\right| p_{\zeta_{x}^{\prime}, C_{x}}\left(x^{\prime}, c\right) \mathrm{d} x^{\prime} .
$$

The probability that a crossing chosen at random will have a speed in the range $\left[\alpha_{1}, \alpha_{2}\right]$ is therefore $N / D$. If we now divide by $\alpha_{2}-\alpha_{1}$ and let both $\alpha_{1}$ and $\alpha_{2}$ tend to a common limit $c$ (so that $\alpha_{2}-\alpha_{1}$ goes to 0 ), we find that the distribution of the speed of the crossing is given by

$$
\tilde{p}_{C_{x}}(c)=\sqrt{\frac{\pi}{2 m_{200}}} \int_{-\infty}^{\infty}\left|x^{\prime}\right| p_{\zeta_{x}^{\prime}, C_{x}}\left(x^{\prime}, c\right) \mathrm{d} x^{\prime},
$$


where

$$
p_{\zeta_{x}^{\prime}, \zeta_{t}^{\prime}}\left(x^{\prime}, t^{\prime}\right)=\frac{\Delta^{-1 / 2}}{2 \pi} \exp \left\{-\frac{1}{2 \Delta}\left(m_{002} x^{\prime 2}-2 m_{101} x^{\prime} t^{\prime}+m_{200} t^{\prime 2}\right)\right\}
$$

with

$$
\Delta=\operatorname{det}\left(\begin{array}{ll}
m_{200} & m_{101} \\
m_{101} & m_{002}
\end{array}\right) \text {. }
$$

Making the change of variable $c=-t^{\prime} / x^{\prime}$, we obtain

$$
\tilde{p}_{C_{x}}(c)=\frac{1}{2} \Delta\left(m_{002}+2 m_{101} c+m_{200} c^{2}\right)^{-3 / 2}\left(m_{200}\right)^{-1 / 2},
$$

which is (with a slightly different notation) Equation (2.5.14) of Longuet-Higgins (1957). As remarked by him, it can also be written as

$$
\tilde{p}_{C_{x}}(c)=\frac{1}{2} \Delta m_{200}^{-2}\left((c-\hat{c})+\Delta m_{200}^{-2}\right)^{-3 / 2}
$$

showing that this distribution is symmetric about its mean value $\hat{c}=-m_{101} / m_{200}$. An important point is that this speed does not depend on the level.

\subsection{Velocity of level curves}

To define the normal velocity of a level curve, we consider the heuristic arguments of Longuet-Higgins (1957). Consider locally a level curve that passes through the point $P$ at time $t$ (see Figure 1). At time $t+\mathrm{d} t$ it passes through $Q$ and $R$ on the $x$ and $y$ axes, respectively, with $\|\overrightarrow{P Q}\|=C_{x} \mathrm{~d} t$ and $\|\overrightarrow{P R}\|=C_{y} \mathrm{~d} t$, where $C_{\underline{x}}$ and $C_{y}$ are defined as in (10).

The velocity of the curve is defined as the vector $\overrightarrow{P T} / \mathrm{d} t$, where $T$ is the orthogonal projection of $P$ onto $\overrightarrow{R Q}$. Let $U$ and $V$ be the projections of $T$ onto the two axes. Elementary trigonometry gives

$$
\overrightarrow{P T}=\frac{C_{x} C_{y}^{2}}{C_{x}^{2}+C_{y}^{2}} \mathrm{~d} t \boldsymbol{e}_{1}+\frac{C_{y} C_{x}^{2}}{C_{x}^{2}+C_{y}^{2}} \mathrm{~d} t \boldsymbol{e}_{2},
$$

where $\boldsymbol{e}_{1}$ is a unit vector along the $x$ axis and $\boldsymbol{e}_{2}$ a unit vector along the $y$ axis, and the velocity of the contour $C_{Q}^{t}(u)=\{(x, y) \in Q: \zeta(x, y, t)=u\}$ at the point $(x, y, t)$ is defined as the vector

$$
\left(\frac{C_{x} C_{y}^{2}}{C_{x}^{2}+C_{y}^{2}}, \frac{C_{y} C_{x}^{2}}{C_{x}^{2}+C_{y}^{2}}\right)
$$

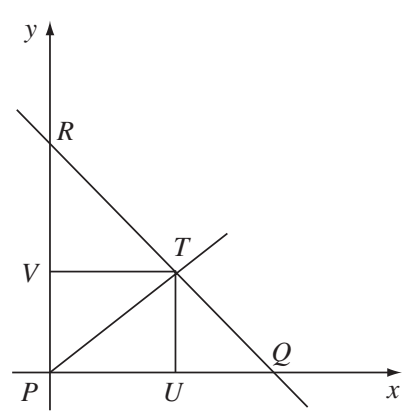

Figure 1: The level curve. 
Following Longuet-Higgins, it is simpler to first obtain the distribution of $\left(K_{x}, K_{y}\right)$, with $K_{x}=\left(C_{x}\right)^{-1}$ and $K_{y}=\left(C_{y}\right)^{-1}$, and then pass to the distribution of the velocity by the change of variable formula. As in Section 5.1, we consider two intervals, $\left[\alpha_{1}, \alpha_{2}\right]$ and $\left[\alpha_{3}, \alpha_{4}\right]$, for $t=0$ and define

$$
D^{\prime}=\mathrm{E}\left[\mathcal{L}\left(C_{Q}(u)\right)\right]=|Q| p(u) \mathrm{E}\left\|\nabla_{12} \zeta(0,0,0)\right\|,
$$

where $\nabla_{12}$ is the gradient limited to the variables $x$ and $y$, and

$$
N^{\prime}=\mathrm{E}\left[\int_{C_{Q}(u)} \mathbf{1}_{\left\{K_{x} \in\left[\alpha_{1}, \alpha_{2}\right]\right\}} \mathbf{1}_{\left\{K_{y} \in\left[\alpha_{3}, \alpha_{4}\right]\right\}} \mathrm{d} \sigma\right] .
$$

This expression is of the form (4) since the integrand in (11) can be written as $\langle\boldsymbol{v}, \boldsymbol{n}\rangle$ with $\boldsymbol{v}=\mathbf{1}_{\left\{K_{x} \in\left[\alpha_{1}, \alpha_{2}\right]\right\}} \mathbf{1}_{\left\{K_{y} \in\left[\alpha_{3}, \alpha_{4}\right]\right\}} \boldsymbol{n}$. Hence,

$$
\begin{aligned}
N^{\prime} & =|Q| p(u) \mathrm{E}\left[\left\|\nabla_{12} \zeta\right\| \mathbf{1}_{\left\{K_{x} \in\left[\alpha_{1}, \alpha_{2}\right]\right\}} \mathbf{1}_{\left\{K_{y} \in\left[\alpha_{3}, \alpha_{4}\right]\right\}}\right] \\
& =|Q| p(u) \int_{\boldsymbol{R}^{3}} \sqrt{x^{\prime 2}+y^{\prime 2}} \mathbf{1}_{\left\{-x^{\prime} / t^{\prime} \in\left[\alpha_{1}, \alpha_{2}\right]\right\}} \mathbf{1}_{\left\{-y^{\prime} / t^{\prime} \in\left[\alpha_{3}, \alpha_{4}\right]\right\}} p_{\zeta_{x}^{\prime}, \zeta_{y}^{\prime}, \zeta_{t}^{\prime}}\left(x^{\prime}, y^{\prime}, t^{\prime}\right) \mathrm{d} x^{\prime} \mathrm{d} y^{\prime} \mathrm{d} t^{\prime}
\end{aligned}
$$

Making the changes of variable $k_{x}=-x^{\prime} / t^{\prime}, k_{y}=-y^{\prime} / t^{\prime}$, and $t^{\prime}=t^{\prime}$ with $\mathrm{d} x^{\prime} \mathrm{d} y^{\prime} \mathrm{d} t^{\prime}=$ $t^{\prime 2} \mathrm{~d} k_{x} \mathrm{~d} k_{y} \mathrm{~d} t^{\prime}$, after some calculations we obtain

$$
\begin{aligned}
N^{\prime}= & 4|Q| p(u) \pi^{-2} \Delta_{2}^{-1 / 2} \\
& \times \int_{\alpha_{1}}^{\alpha_{2}} \int_{\alpha_{3}}^{\alpha_{4}} \mathrm{~d} k_{x} \mathrm{~d} k_{y} \sqrt{k_{x}^{2}+k_{y}^{2}} \\
& \quad \times\left(\mu_{11} k_{x}^{2}+2 \mu_{12} k_{x} k_{y}-2 \mu_{13} k_{x}+\mu_{22} k_{y}^{2}-2 \mu_{23} k_{y}+\mu_{33}\right)^{-2},
\end{aligned}
$$

where $\Delta_{2}$ and $\mu_{i j}$ are, respectively, the determinant and the entries of the inverse matrix of

$$
\left(\begin{array}{lll}
m_{200} & m_{110} & m_{101} \\
m_{110} & m_{020} & m_{011} \\
m_{101} & m_{011} & m_{002}
\end{array}\right)
$$

Letting $\alpha_{1}$ and $\alpha_{2}$ tend to $k_{x}$ and $\alpha_{3}$ and $\alpha_{4}$ tend to $k_{y}$, we find that the distribution of $K_{x}$ and $K_{y}$ is

$$
\begin{aligned}
\tilde{p}_{k_{x}, k_{y}}= & \lim _{\substack{\alpha_{1}, \alpha_{2} \rightarrow k_{x} \\
\alpha_{3}, \alpha_{4} \rightarrow k_{y}}} \frac{1}{\left(\alpha_{2}-\alpha_{1}\right)\left(\alpha_{4}-\alpha_{3}\right)} \frac{N^{\prime}}{D^{\prime}} \\
= & \frac{1}{\pi}\left(\gamma_{2}\right)^{-1 / 2} \Delta_{2}^{-1 / 2}(\mathcal{E}(k))^{-1} \sqrt{k_{x}^{2}+k_{y}^{2}} \\
& \times\left(\mu_{11} k_{x}^{2}+2 \mu_{12} k_{x} k_{y}-2 \mu_{13} k_{x}+\mu_{22} k_{y}^{2}-2 \mu_{23} k_{y}+\mu_{33}\right)^{-2},
\end{aligned}
$$

where $k=\left(1-\gamma_{1} / \gamma_{2}\right)^{1 / 2}$ as before, but $\gamma_{1}$ and $\gamma_{2}$ are the eigenvalues of the matrix

$$
\left(\begin{array}{ll}
m_{200} & m_{110} \\
m_{110} & m_{020}
\end{array}\right)
$$

With this, we have again recovered a result of Longuet-Higgins (1957) (Equation 2.6.21, in fact) and we again remark that the result does not depend on the level $u$. 
We now look at the distribution of the velocity

$$
\boldsymbol{V}=\left(V_{x}, V_{y}\right)=\left(\frac{k_{x}}{k_{x}^{2}+k_{y}^{2}}, \frac{k_{y}}{k_{x}^{2}+k_{y}^{2}}\right) ;
$$

we have $k_{x}=V_{x} /\left(v_{x}^{2}+v_{y}^{2}\right), k_{y}=V_{y} /\left(v_{x}^{2}+v_{y}^{2}\right)$, and $\mathrm{d} k_{x} \mathrm{~d} k_{y}=\left(V_{x}^{2}+V_{y}^{2}\right)^{-2} \mathrm{~d} V_{x} \mathrm{~d} V_{y}$. As a consequence,

$$
\begin{aligned}
\tilde{p}_{V_{x}, V_{y}}= & \frac{1}{\pi}\left(\gamma_{2}\right)^{-1 / 2} \Delta_{2}^{-1 / 2}(\mathcal{E}(k))^{-1}\left(V_{x}^{2}+V_{y}^{2}\right)^{7 / 2} \\
& \times\left(\mu_{11} V_{x}^{2}+2 \mu_{12} V_{x} V_{y}-2 \mu_{13} V_{x}\left(V_{x}^{2}+V_{y}^{2}\right)\right. \\
& \left.\quad+\mu_{22} V_{y}^{2}-2 \mu_{23} V_{y}\left(V_{x}^{2}+V_{y}^{2}\right)+\mu_{33}\left(V_{x}^{2}+V_{y}^{2}\right)^{2}\right)^{-2} .
\end{aligned}
$$

Remark 3. (Velocity of crests.) Since the distributions of $\zeta$ and $-\zeta$ are the same, the mean velocity of a crest is the mean velocity of the zero level set for the process $\zeta_{\theta}^{\prime}$. Thus, the same kind of result holds if we change the meaning of the moments in matrix (12).

\section{Chaos expansion and central limit theorem}

In this section, we develop the Itô-Wiener chaos expansion for the length of the static crest considered in Section 4, and apply it to obtain the central limit theorem for the length of the crest. In what follows, we will assume that this level functional belongs to $L^{2}(\Omega)$, which property holds under several hypotheses given, for example, in Cabaña (1985, p. 79) and Wschebor (1985, p. 54).

We begin by transforming $\zeta_{\theta}^{\prime}(x, y)$ into an isotropic random field. This step is necessary to obtain the chaos development of the level functionals. We can assume, without loss of generality, that $\mathrm{E}\left[\left(\zeta_{\theta}^{\prime}(x, y)\right)^{2}\right]=1$. As in Section 4 , we consider the matrix $\Sigma(\theta)$, but we will drop explicit reference to its argument $\theta$, for ease of notation. Define

$$
\eta_{\theta}(x, y)=\zeta_{\theta}^{\prime}\left[\Sigma^{-1 / 2}\left(\begin{array}{l}
x \\
y
\end{array}\right)\right]
$$

it is easy to check that $\eta_{\theta}(x, y)$ is an isotropic random field. Considering formula (5) for the field $\zeta_{\theta}^{\prime}(x, y)$ and taking $\gamma(\varphi(x, y))=\mathbf{1}_{\{\cos (\varphi(x, y))<0\}}$, we have

$$
\begin{aligned}
& \int_{\mathbb{R}} g(u) \mathrm{d} u \int_{\left\{(x, y) \in Q(N): \zeta_{\theta}^{\prime}(x, y)=u\right\}} \gamma(\varphi) \mathrm{d} \sigma \\
& \quad=\iint_{Q(N)} g\left(\zeta_{\theta}^{\prime}(x, y)\right) \mathbf{1}_{\{\cos (\varphi(x, y))<0\}}\left\|\nabla \zeta_{\theta}^{\prime}(x, y)\right\| \mathrm{d} x \mathrm{~d} y,
\end{aligned}
$$

where $Q(N)=[-N b, N b] \times[-N b, N b]$ for a fixed $b>0$. When $u=0$, the second integral on the left-hand side corresponds to the length of the crest, and the right-hand side is

$$
\begin{aligned}
& \iint_{Q(N)} g\left(\zeta_{\theta}^{\prime}(x, y)\right) \mathbf{1}_{(-\infty, 0)}\left(\zeta_{\theta \theta}^{\prime \prime}(x, y)\right)\left\|\nabla \zeta_{\theta}^{\prime}(x, y)\right\| \mathrm{d} x \mathrm{~d} y \\
& =\iint_{Q(N)} g\left(\eta_{\theta}\left[\Sigma^{1 / 2}\left(\begin{array}{l}
x \\
y
\end{array}\right)\right]\right) \mathbf{1}_{(-\infty, 0)}\left(b_{11} \partial_{x} \eta_{\theta}\left[\Sigma^{1 / 2}\left(\begin{array}{l}
x \\
y
\end{array}\right)\right]+b_{12} \partial_{y} \eta_{\theta}\left[\Sigma^{1 / 2}\left(\begin{array}{l}
x \\
y
\end{array}\right)\right]\right) \\
& \quad \times\left\|\Sigma^{1 / 2} \nabla \eta_{\theta}\left[\Sigma^{1 / 2}\left(\begin{array}{l}
x \\
y
\end{array}\right)\right]\right\| \mathrm{d} x \mathrm{~d} y,
\end{aligned}
$$


where $b_{i j}$ are the entries of $\Sigma^{1 / 2}$. Making the change of variables

$$
\Sigma^{1 / 2}\left(\begin{array}{l}
x \\
y
\end{array}\right)=\left(\begin{array}{l}
x^{\prime} \\
y^{\prime}
\end{array}\right)
$$

in the last double integral above, we find that (13) is equal to

$$
\begin{aligned}
|\boldsymbol{\Sigma}|^{-1 / 2} \iint_{S(N)} & g\left(\eta_{\theta}\left(x^{\prime}, y^{\prime}\right)\right) \mathbf{1}_{(-\infty, 0)}\left(b_{11} \partial_{x} \eta_{\theta}\left(x^{\prime}, y^{\prime}\right)+b_{12} \partial_{y} \eta_{\theta}\left(x^{\prime}, y^{\prime}\right)\right) \\
& \times\left\|\boldsymbol{\Sigma}^{1 / 2} \nabla \eta_{\theta}\left(x^{\prime}, y^{\prime}\right)\right\| \mathrm{d} x^{\prime} \mathrm{d} y^{\prime}
\end{aligned}
$$

where $S(N)=\Sigma^{1 / 2}(Q(N))$.

Let us explain the heuristics of our development, for which we introduce the following level functional, where $\phi$ is the standard normal density:

$$
\begin{aligned}
\mathcal{L}_{\sigma}(u) & =\frac{1}{\sigma} \int_{-\infty}^{\infty} \phi\left(\frac{u-v}{\sigma}\right)\left(\int_{\left\{(x, y) \in Q(N): \zeta_{\theta}^{\prime}(x, y)=v\right\}} \gamma(\varphi(s)) \mathrm{d} \sigma(s)\right) \mathrm{d} v \\
& =\frac{1}{\sigma} \iint_{Q(N)} \phi\left(\frac{u-\zeta_{\theta}^{\prime}(x, y)}{\sigma}\right) \gamma(\varphi(x, y))\left\|\nabla \zeta_{\theta}^{\prime}(x, y)\right\| \mathrm{d} x \mathrm{~d} y .
\end{aligned}
$$

By using the same change of variables as above, this random variable is equal to

$$
\begin{aligned}
\frac{|\boldsymbol{\Sigma}|^{1 / 2}}{\sigma} \iint_{S(N)} & \phi\left(\frac{u-\eta_{\theta}\left(x^{\prime}, y^{\prime}\right)}{\sigma}\right) \mathbf{1}_{(\infty, 0)}\left(b_{11} \partial_{x} \eta_{\theta}\left(x^{\prime}, y^{\prime}\right)+b_{12} \partial_{y} \eta_{\theta}\left(x^{\prime}, y^{\prime}\right)\right) \\
& \times\left\|\Sigma^{1 / 2} \nabla \eta_{\theta}\left(x^{\prime}, y^{\prime}\right)\right\| \mathrm{d} x^{\prime} \mathrm{d} y^{\prime} .
\end{aligned}
$$

Let $D^{2} r_{\theta}(x, y)$ be the matrix of second derivative of $r_{\theta}(x, y)$, the covariance of the process $\zeta_{\theta}^{\prime}$. It can be shown that this functional converges in $L^{2}(\Omega)$, under the hypothesis $D^{2} r_{\theta}(0,0)-$ $D^{2} r_{\theta}(x, y) \neq 0$, to a functional that, when $u=0$, is the length of the crest. (For a proof in the case of the length of the level curve, see Kratz and León (2001). The condition on the derivatives is given in Berzin and Wschebor (1993). Our case could be treated in a similar fashion.) Hence, obtaining a chaos expansion for $\mathcal{L}_{\sigma}(u)$ implies an analogous expansion for $\mathcal{L}\left(C_{Q, \theta}^{\mathrm{s}}\right)$; let us first obtain the chaos expansion for $\mathcal{L}_{\sigma}(u)$. Using the expansions in $L^{2}(\phi(v) \mathrm{d} v)$, we get

$$
\frac{1}{\sigma} \phi\left(\frac{u-v}{\sigma}\right)=\sum_{l=0}^{\infty} c_{l}(u, \sigma) H_{l}(v) \quad \text { with } \quad c_{l}(u, \sigma)=\frac{1}{\sigma l !} \int_{-\infty}^{\infty} \phi\left(\frac{u-v}{\sigma}\right) H_{l}(v) \phi(v) \mathrm{d} v
$$

where $H_{l}$ is the Hermite polynomial of order $l$. Now consider the decomposition $\Sigma^{1 / 2}=$ $\boldsymbol{P} \boldsymbol{D}^{1 / 2} \boldsymbol{P}^{-1}$ where $\boldsymbol{D}$ is a diagonal matrix with eigenvalues $\gamma_{i}$ and $\boldsymbol{P}$, defined as

$$
\boldsymbol{P}=\left(\begin{array}{cc}
\cos \alpha_{0} & -\sin \alpha_{0} \\
\sin \alpha_{0} & \cos \alpha_{0}
\end{array}\right)
$$

is orthogonal. We have

$$
\begin{aligned}
& \mathbf{1}_{(\infty, 0)}\left(b_{11} \partial_{x} \eta_{\theta}\left(x^{\prime}, y^{\prime}\right)+b_{12} \partial_{y} \eta_{\theta}\left(x^{\prime}, y^{\prime}\right)\right)\left\|\boldsymbol{\Sigma}^{1 / 2} \nabla \eta_{\theta}\left(x^{\prime}, y^{\prime}\right)\right\| \mathrm{d} x^{\prime} \mathrm{d} y^{\prime} \\
& =\mathbf{1}_{(\infty, 0)}\left(\left\langle\boldsymbol{P} D^{1 / 2}\left[\boldsymbol{P}^{-1} \nabla \eta_{\theta}\left(x^{\prime}, y^{\prime}\right)\right], \boldsymbol{e}_{1}\right\rangle\right)\left\|\boldsymbol{P} \boldsymbol{D}^{1 / 2}\left[\boldsymbol{P}^{-1} \nabla \eta_{\theta}\left(x^{\prime}, y^{\prime}\right)\right]\right\| \mathrm{d} x^{\prime} \mathrm{d} y^{\prime} .
\end{aligned}
$$


The Gaussian vector

$$
\boldsymbol{P}^{-1} \nabla \eta_{\theta}\left(x^{\prime}, y^{\prime}\right)=\left(\begin{array}{c}
\partial_{x^{\prime}} \eta_{\theta} \cos \alpha_{0}+\partial_{y^{\prime}} \eta_{\theta} \sin \alpha_{0} \\
-\partial_{x^{\prime}} \eta_{\theta} \sin \alpha_{0}+\partial_{y^{\prime}} \eta_{\theta} \cos \alpha_{0}
\end{array}\right)
$$

is standard for each fixed $\left(x^{\prime}, y^{\prime}\right)$. Using the expansions in $L^{2}(\phi(x) \phi(y) \mathrm{d} x \mathrm{~d} y)$, we find that (14) is equal to

$$
\sum_{m, n} d_{m, n} H_{m}\left(\partial_{x^{\prime}} \eta_{\theta} \cos \alpha_{0}+\partial_{y^{\prime}} \eta_{\theta} \sin \alpha_{0}\right) H_{n}\left(-\partial_{x^{\prime}} \eta_{\theta} \sin \alpha_{0}+\partial_{y^{\prime}} \eta_{\theta} \cos \alpha_{0}\right),
$$

where

$$
\begin{aligned}
& d_{m, n}=\frac{1}{n ! m !} \int_{\mathbb{R}^{2}} \sqrt{\gamma_{1} x^{2}+\gamma_{2} y^{2}} \\
& \times \mathbf{1}_{(-\infty, 0)}\left(\sqrt{\gamma_{1}} x \cos \alpha_{0}-\sqrt{\gamma_{2}} y \sin \alpha_{0}\right) H_{m}(x) H_{n}(y) \phi(x) \phi(y) \mathrm{d} x \mathrm{~d} y \\
&=\frac{1}{2 \pi n ! m !} \int_{0}^{\infty} \int_{0}^{2 \pi} \sqrt{\gamma_{1} \cos ^{2} \xi+\gamma_{2} \sin ^{2} \xi} \\
& \quad \times \mathbf{1}_{(-\infty, 0)}(\cos (\delta+\xi)) H_{m}(\rho \cos \xi) H_{n}(\rho \sin \xi) \mathrm{e}^{-\rho^{2} / 2} \rho^{2} \mathrm{~d} \rho \mathrm{d} \xi
\end{aligned}
$$

- the latter follows from changing to polar coordinates and using

$$
\delta=\arctan \left(\frac{\sin \alpha_{0}\left(\gamma_{2}\right)^{1 / 2}}{\cos \alpha_{0}\left(\gamma_{1}\right)^{1 / 2}}\right)
$$

Thus,

$$
\begin{aligned}
d_{m, n}=\frac{1}{2 \pi n ! m !} \int_{0}^{\infty} \int_{\pi / 2-\delta}^{3 \pi / 2-\delta} & \sqrt{\gamma_{1} \cos ^{2} \xi+\gamma_{2} \sin ^{2} \xi} \\
& \times H_{m}(\rho \cos \xi) H_{n}(\rho \sin \xi) \mathrm{e}^{-\rho^{2} / 2} \rho^{2} \mathrm{~d} \rho \mathrm{d} \xi
\end{aligned}
$$

If $m$ and $n$ are both even then the function inside the integral is periodic with period $\pi$, and

$$
\begin{aligned}
d_{2 k, 2 l}=\frac{1}{2 \pi(2 k) !(2 l) !} \int_{0}^{\infty} \int_{0}^{\pi} & \sqrt{\gamma_{1} \cos ^{2} \xi+\gamma_{2} \sin ^{2} \xi} \\
& \times H_{2 k}(\rho \cos \xi) H_{2 l}(\rho \sin \xi) \mathrm{e}^{-\rho^{2} / 2} \rho^{2} \mathrm{~d} \rho \mathrm{d} \xi .
\end{aligned}
$$

If both indices are odd then the function is again of period $\pi$ but is an odd function, giving $d_{2 k+1,2 l+1}=0$. The remaining case, when one index is even and the other is odd, is more involved and it cannot be guaranteed that the function inside the integral is of period $\pi$. The integral must be computed for $\xi \in\left[\frac{1}{2} \pi-\delta, \frac{3}{2} \pi-\delta\right]$.

Let us return to the expansion for $\mathcal{L}_{\sigma}(u)$ and define

$$
\begin{aligned}
\ell_{q, m, n}\left(x^{\prime}, y^{\prime}\right)= & H_{q-(m+n)}\left(\eta_{\theta}\right) H_{m}\left(\partial_{x^{\prime}} \eta_{\theta} \cos \alpha_{0}+\partial_{y^{\prime}} \eta_{\theta} \sin \alpha_{0}\right) \\
& \times H_{n}\left(-\partial_{x^{\prime}} \eta_{\theta} \sin \alpha_{0}+\partial_{y^{\prime}} \eta_{\theta} \cos \alpha_{0}\right)
\end{aligned}
$$

and

$$
c_{q, m, n}=\frac{1}{\sqrt{2 \pi}(q-(m+n)) !} H_{q-(m+n)}(0) d_{m, n} .
$$

Using the fact that the random variable

$$
\frac{1}{\sigma} \phi\left(\frac{u-\eta_{\theta}(0,0)}{\sigma}\right) \mathbf{1}_{(\infty, 0)}\left(b_{11} \partial_{x} \eta_{\theta}(0,0)+b_{12} \partial_{y} \eta_{\theta}(0,0)\right)\left\|\Sigma^{1 / 2} \nabla \eta_{\theta}(0,0)\right\|
$$


belongs to $L^{2}(\Omega)$, and since $\mathcal{L}_{\sigma}(u)$ is an additive functional of the field and the different terms in the chaos expansion are orthogonal, we readily find that

$$
\mathcal{L}_{\sigma}(u)=|\Sigma|^{-1 / 2} \sum_{q=0}^{\infty} \sum_{0 \leq m+n \leq q} c_{q-(m+n)}(u, \sigma) d_{m, n} \iint_{S(N)} \ell_{q, m, n}\left(x^{\prime}, y^{\prime}\right) \mathrm{d} x^{\prime} \mathrm{d} y^{\prime} .
$$

However,

$$
\lim _{\sigma \rightarrow 0} c_{l}(u, \sigma)=\frac{1}{l !} H_{l}(u) \phi(u),
$$

so by letting $\sigma$ tend to 0 , setting $u=0$, and using the convergence in $L^{2}(\Omega)$, we obtain the following result.

Proposition 1. Assume that $\mathcal{L}\left(C_{Q, \theta}^{\mathrm{s}}\right)$ belongs to $L^{2}(\Omega)$ and that $D^{2} r_{\theta}(0,0)-D^{2} r_{\theta}(x, y) \neq 0$. We then have the following expansion for the length of the crest:

$$
\mathcal{L}\left(C_{Q, \theta}^{\mathrm{s}}\right)=|\boldsymbol{\Sigma}|^{-1 / 2} \sum_{q=0}^{\infty} \sum_{0 \leq m+n \leq q} c_{q, m, n} \iint_{S(T)} \ell_{q, m, n}\left(x^{\prime}, y^{\prime}\right) \mathrm{d} x^{\prime} \mathrm{d} y^{\prime} .
$$

Using this expansion, we can compute the expectation of this random variable, obtaining

$$
\mathrm{E}\left[\mathcal{L}\left(C_{Q, \theta}^{\mathrm{s}}\right)\right]=\frac{1}{\sqrt{2 \pi}} d_{0,0}|Q(N)|
$$

Since $d_{0,0}=\left(\gamma_{2} / 2 \pi\right)^{1 / 2} \mathcal{E}(k)$, this coincides with our previous result of Section 3 .

We are interested in studying the central limit theorem for the functional $\mathcal{L}\left(C_{Q, \theta}^{\mathrm{s}}\right)$. First we must ensure that the law of large numbers holds, which will be a consequence of the ergodic theorem. Given that the process is Gaussian, it is ergodic if the covariance

$$
r_{\theta}(x, y)=\mathrm{E}\left[\zeta_{\theta}^{\prime}(x, y) \zeta_{\theta}^{\prime}(0,0)\right]
$$

tends to 0 as $\left(x^{2}+y^{2}\right)^{1 / 2} \rightarrow \infty$. Thus, under this hypothesis we have

$$
\frac{\mathcal{L}\left(C_{Q, \theta}^{\mathrm{s}}\right)}{|Q(N)|} \rightarrow \frac{\sqrt{\gamma_{2}}}{2 \pi} \mathcal{E}(k)
$$

almost surely as $|Q(N)| \rightarrow \infty$.

We will study the weak limit of the random variable

$$
\begin{aligned}
w & :=\sqrt{|Q(N)|}\left(\frac{\mathcal{L}\left(C_{Q, \theta}^{\mathrm{s}}\right)}{|Q(N)|}-\frac{\sqrt{\gamma_{2}}}{2 \pi} \mathcal{E}(k)\right) \\
& =\frac{1}{\sqrt{|S(N)|}} \sum_{q=1}^{\infty} \sum_{0 \leq m+n \leq q} c_{q, m, n} \iint_{S(N)} \iota_{q, m, n}\left(x^{\prime}, y^{\prime}\right) \mathrm{d} x^{\prime} \mathrm{d} y^{\prime}
\end{aligned}
$$


by first computing its variance. Given the orthogonality of the different chaos expansions, we have

$$
\begin{aligned}
& |Q(N)| \mathrm{E}\left[\frac{\mathcal{L}\left(C_{Q, \theta}^{\mathrm{s}}\right)}{|Q(N)|}-\frac{\sqrt{\gamma_{2}}}{2 \pi} \&(k)\right]^{2} \\
& =\frac{1}{|S(N)|} \sum_{q=1}^{\infty} \mathrm{E}\left[\sum_{0 \leq m+n \leq q} c_{q, m, n} \iint_{S(N)} \ell_{q, m, n}\left(x^{\prime}, y^{\prime}\right) \mathrm{d} x^{\prime} \mathrm{d} y^{\prime}\right]^{2} \\
& =\frac{1}{|S(N)|} \sum_{q=1}^{\infty} \int_{S(N) \times S(N)} \mathrm{E}\left[\mathcal{F}_{q}\left(\boldsymbol{x}^{\prime}\right) \mathcal{F}_{q}\left(\boldsymbol{x}^{\prime \prime}\right)\right] \mathrm{d} \boldsymbol{x}^{\prime} \mathrm{d} \boldsymbol{x}^{\prime \prime}
\end{aligned}
$$

where $\boldsymbol{x}^{\prime}=\left(x^{\prime}, y^{\prime}\right)^{\top}, \boldsymbol{x}^{\prime \prime}=\left(x^{\prime \prime}, y^{\prime \prime}\right)^{\top}$, and

$$
\mathcal{F}_{q}\left(\boldsymbol{x}^{\prime}\right)=\sum_{0 \leq m+n \leq q} c_{q, m, n} \iota_{q, m, n}\left(x^{\prime}, y^{\prime}\right) .
$$

Making the changes of variable $\boldsymbol{x}^{\prime}=\Sigma^{1 / 2} \boldsymbol{u}^{\prime}$ and $\boldsymbol{x}^{\prime \prime}=\Sigma^{1 / 2} \boldsymbol{u}^{\prime \prime}$ in (16), we find that it becomes

$$
\frac{1}{|Q(N)|} \sum_{q=1}^{\infty} \int_{Q(N) \times Q(N)} \mathrm{E}\left[\mathcal{F}_{q}\left(\Sigma^{1 / 2} \boldsymbol{u}^{\prime}\right) \mathcal{F}_{q}\left(\Sigma^{1 / 2} \boldsymbol{u}^{\prime \prime}\right)\right] \mathrm{d} \boldsymbol{u}^{\prime} \mathrm{d} \boldsymbol{u}^{\prime \prime}
$$

Let us suppose that $Q(N)=[-N, N] \times[-N, N]$. We can decompose this region as

$$
Q(N)=\bigcup_{i, j=-N+1}^{N} Q_{i j}
$$

where $Q_{i j}=((i-1), i] \times((j-1), j]$. Thus, for (17) we have

$$
\frac{1}{N^{2}} \sum_{q=1}^{\infty} \sum_{i, j=-N+1}^{N} \sum_{i^{\prime}, j^{\prime}=-N+1}^{N} \int_{Q_{i j} \times Q_{i^{\prime} j^{\prime}}} \mathrm{E}\left[\mathcal{F}_{q}\left(\Sigma^{1 / 2} \boldsymbol{u}^{\prime}\right) \mathcal{F}_{q}\left(\Sigma^{1 / 2} \boldsymbol{u}^{\prime \prime}\right)\right] \mathrm{d} \boldsymbol{u}^{\prime} \mathrm{d} \boldsymbol{u}^{\prime \prime}
$$

By further defining

$$
Y_{00}=\sum_{q=1}^{\infty} \int_{Q_{11}} \mathcal{F}_{q}\left(\Sigma^{1 / 2} \boldsymbol{u}\right) \mathrm{d} \boldsymbol{u}
$$

and using the bidimensional shift associated with the field $\zeta$, defined as

$$
\Gamma_{s, t} \zeta(\cdot, \cdot)=\zeta(\cdot+s, \cdot+t)
$$

we can write (18) as

$$
\frac{(2 N+1)^{2}}{N^{2}} \sum_{i=-N+1}^{N} \sum_{j=-N+1}^{N}\left(1-\frac{|i|}{2 N+1}\right)\left(1-\frac{|j|}{2 N+1}\right) \mathrm{E}\left[Y_{00} \Gamma_{i, j}\left(Y_{00}\right)\right] .
$$

We shall assume, for simplicity, the very strong hypothesis that the field $\zeta$ is $a$-dependent, i.e. the correlation function $r(x, y)$ vanishes when $\left(x^{2}+y^{2}\right)^{1 / 2}>a$. Under this condition, we 
find that

$$
\begin{aligned}
\lim _{N \rightarrow \infty} & \frac{(2 N+1)^{2}}{N^{2}} \sum_{i=-N+1}^{N} \sum_{j=-N+1}^{N}\left(1-\frac{|i|}{2 N+1}\right)\left(1-\frac{|j|}{2 N+1}\right) \mathrm{E}\left[Y_{00} \Gamma_{i, j}\left(Y_{00}\right)\right] \\
& =\sum_{i=-a}^{a} \sum_{j=-a}^{a} \mathrm{E}\left[Y_{00} \Gamma_{i, j}\left(Y_{00}\right)\right]=\sum_{q=1}^{\infty} \int_{\left\|\Sigma^{1 / 2} \boldsymbol{u}^{\prime}\right\| \leq a} \mathrm{E}\left[\mathcal{F}_{q}\left(\Sigma^{1 / 2} \boldsymbol{u}^{\prime}\right) \mathcal{F}_{q}(0)\right] \mathrm{d} \boldsymbol{u}^{\prime} \\
& =\sum_{q=1}^{\infty} \sigma_{q}^{2}<\infty
\end{aligned}
$$

It is now plain how to obtain the central limit theorem. The result established above allows us to approximate $w$ (see $(15))$ in $L^{2}(\Omega)$ by a finite expansion, i.e.

$$
\begin{aligned}
Z_{Q} & =\frac{1}{\sqrt{|S(N)|}} \sum_{q=1}^{Q} \sum_{0 \leq m+n \leq q} c_{q, m, n} \iint_{S(N)} \ell_{q, m, n}\left(x^{\prime}, y^{\prime}\right) \mathrm{d} x^{\prime} \mathrm{d} y^{\prime} \\
& =\frac{1}{\sqrt{|S(N)|}} \iint_{S(N)} f_{Q}\left(x^{\prime}, y^{\prime}\right) \mathrm{d} x^{\prime} \mathrm{d} y^{\prime}
\end{aligned}
$$

where

$$
f_{Q}\left(x^{\prime}, y^{\prime}\right)=\sum_{q=1}^{Q} \sum_{0 \leq m+n \leq q} c_{q, m, n} \iota_{q, m, n}\left(x^{\prime}, y^{\prime}\right) .
$$

The weak convergence of the latter sequence to a Gaussian random variable is a direct consequence of the central limit theorem for $a$-dependent sums.

In summary, we have proved the following theorem.

Theorem 1. If the Gaussian field $\zeta_{\theta}^{\prime}(x, y)$ is a-dependent and the level functional $\mathcal{L}\left(C_{Q, \theta}^{\mathrm{s}}\right)$ belongs to $L^{2}(\Omega)$, then the weak limit of

$$
\sqrt{|Q(N)|}\left(\frac{\mathcal{L}\left(C_{Q, \theta}^{\mathrm{s}}\right)}{|Q(N)|}-\frac{\sqrt{\gamma_{2}}}{2 \pi} \mathcal{E}(k)\right)
$$

as $N \rightarrow \infty$ is centered normal with variance $\sum_{q=1}^{\infty} \sigma_{q}^{2}$.

Remarks 4. One of the advantages of the development in chaos is that we can show that the limit variance is strictly positive. To do so, it is enough to show that $\sigma_{q}^{2}>0$ for some value of $q$. This is easy to do for $q=2$ because all the associated terms vanish except for the one corresponding to $c_{2,0,0}$. In this case, the only polynomial appearing is $\mathrm{H}_{2}$.

It is possible to weaken the strong dependence hypothesis we have used, requiring, for example, $L^{1}$ - and $L^{2}$-integrability of all covariances involved and no other mixing condition, but the proof in such a case is rather cumbersome and involves tedious computations. For the case of contours, this was fully developed in Kratz and León (2001).

We can also study the crest above a certain level $h$. For this, we consider the set

$$
\mathcal{C}_{Q, \theta}^{\mathrm{s}}(h)=\left\{(x, y) \in Q: \zeta_{\theta}^{\prime}(x, y)=0, \zeta_{\theta}^{\prime \prime}(x, y)<0, \zeta(x, y) \geq h\right\} .
$$

To compute the length of this set, let us consider the vector field

$$
\boldsymbol{v}=\mathbf{1}_{[h, \infty)}(\zeta(x, y)) \mathbf{1}_{(-\infty, 0)}(\cos \varphi(x, y)) \boldsymbol{n} .
$$


Using formula (3), we obtain

$$
\begin{aligned}
\int_{R} g(u) \mathrm{d} u \int_{\left\{(x, y) \in Q: \zeta_{\theta}^{\prime}(x, y)=u, \zeta_{\theta}^{\prime \prime}(x, y)<0, \zeta(x, y) \geq h\right\}}\langle\boldsymbol{v}, \boldsymbol{n}\rangle \mathrm{d} \sigma \\
=\iint_{Q(N)} \mathbf{1}_{[h, \infty)}(\zeta(x, y)) g\left(\zeta_{\theta}^{\prime}(x, y)\right)\left\|\nabla \zeta_{\theta}^{\prime}(x, y)\right\| \mathrm{d} x \mathrm{~d} y .
\end{aligned}
$$

In the same manner as we have done for the crest, we can obtain

$$
\mathcal{L}\left(\mathcal{C}_{Q, \theta}^{\mathrm{s}}(h)\right)=\lim _{\sigma \rightarrow 0} \frac{1}{\sigma} \iint_{Q(N)} \mathbf{1}_{[h, \infty)}(\zeta(x, y)) \phi\left(\frac{\zeta_{\theta}^{\prime}(x, y)}{\sigma}\right)\left\|\nabla \zeta_{\theta}^{\prime}(x, y)\right\| \mathrm{d} x \mathrm{~d} y .
$$

This limit is in $L^{2}(\Omega)$. To obtain the chaos development, we must follow the same procedure as for the simple crest, but this is now more involved, due to the fact that the random variable $\zeta(x, y)$ is not independent of the random vector $\nabla \zeta_{\theta}^{\prime}(x, y)$.

\section{Real data}

In this section, we consider two directional spectra kindly provided by M. Prevosto from Ifremer (the French Research Institute for Exploration of the Sea). These are depicted in Figure 2.

Figure 3 shows the level curves for the spectra.

Figure 4 shows the integrals of the spectra along fixed directions, measured in degrees, showing a peak at around $165^{\circ}$. The integrals are calculated over the half-line starting at the origin.

Figures 2, 3, and 4 show that the energy is concentrated around the $165^{\circ}$ direction, which is understood to be the most probable direction for the waves.

Figure 5 shows the expected length of crests along directions spaced by $15^{\circ}$, showing a maximum (in the static case) at approximately $75^{\circ}$. It is interesting to observe that, in accordance with theoretical results, this direction is orthogonal to the direction for the maximum integral of the spectrum, i.e. is the most probable direction for the waves.

Figure 6 shows the level curves for probability densities of the velocity. Both graphs show a clear asymmetry, as predicted by Longuet-Higgins (1957).

For colour versions of the figures, see http://www.lsp.ups-tlse.fr/Azais/publi/olas7.ps.
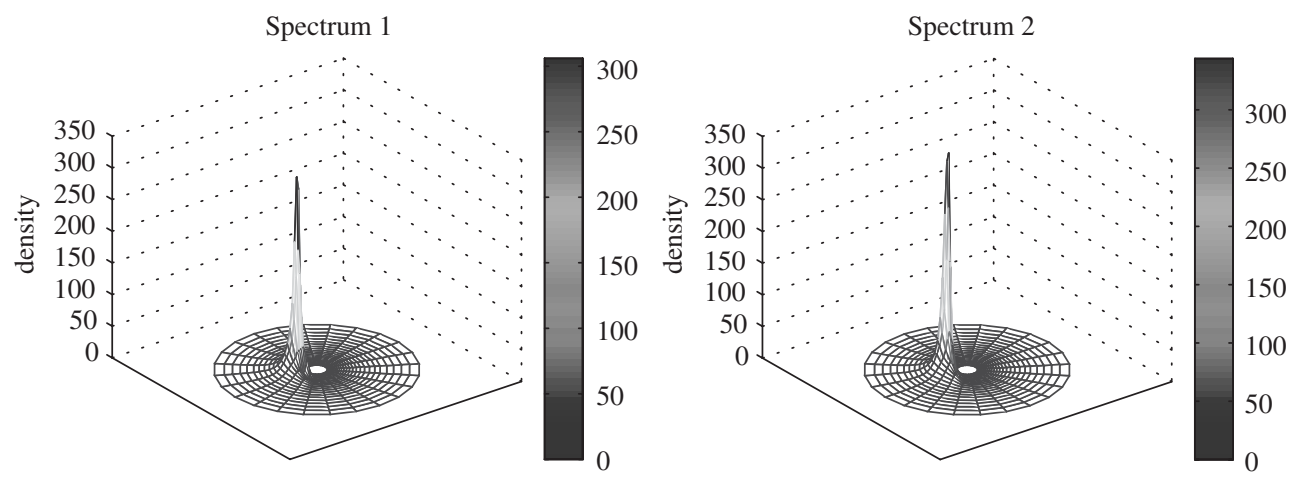

FIGURE 2: The directional spectra. 
Level curves for spectrum 1

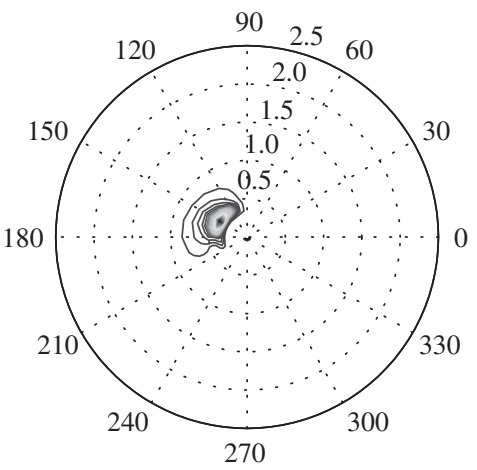

Level curves for spectrum 2

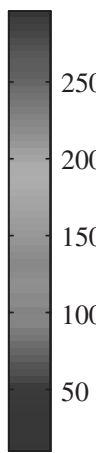

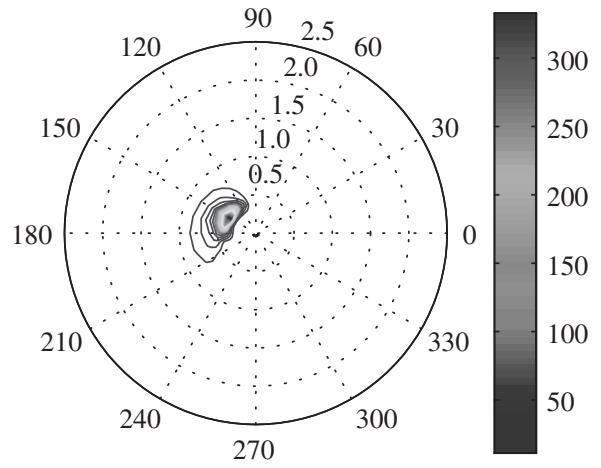

Figure 3: The level curves.

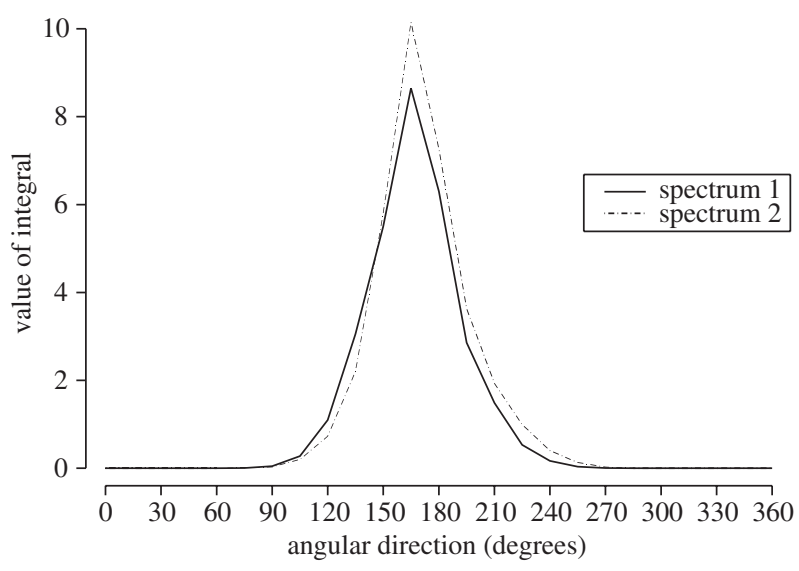

FIGURE 4: Spectrum integrals along fixed directions.
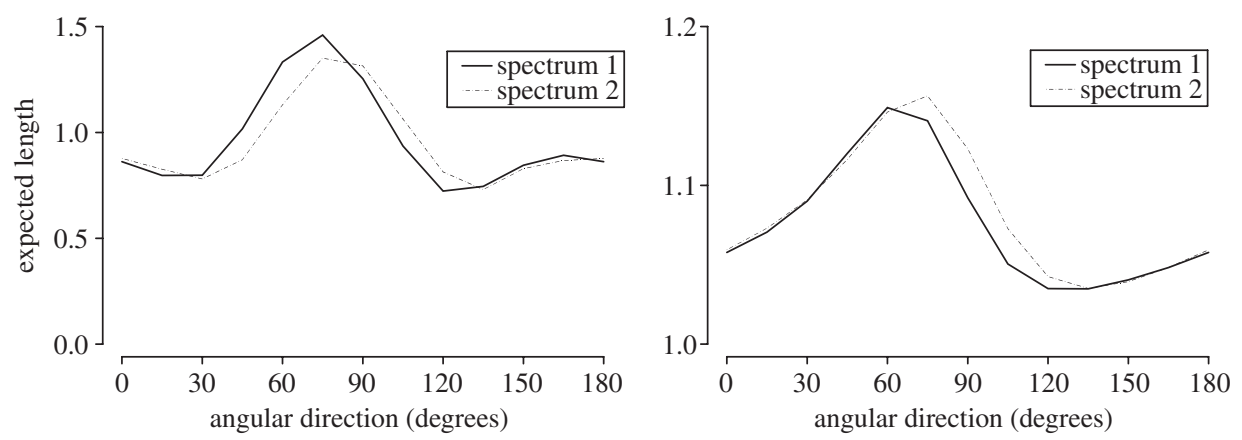

FIGURE 5: Expected length of crests per unit area along fixed directions in (left) the static case and (right) the dynamic case. 

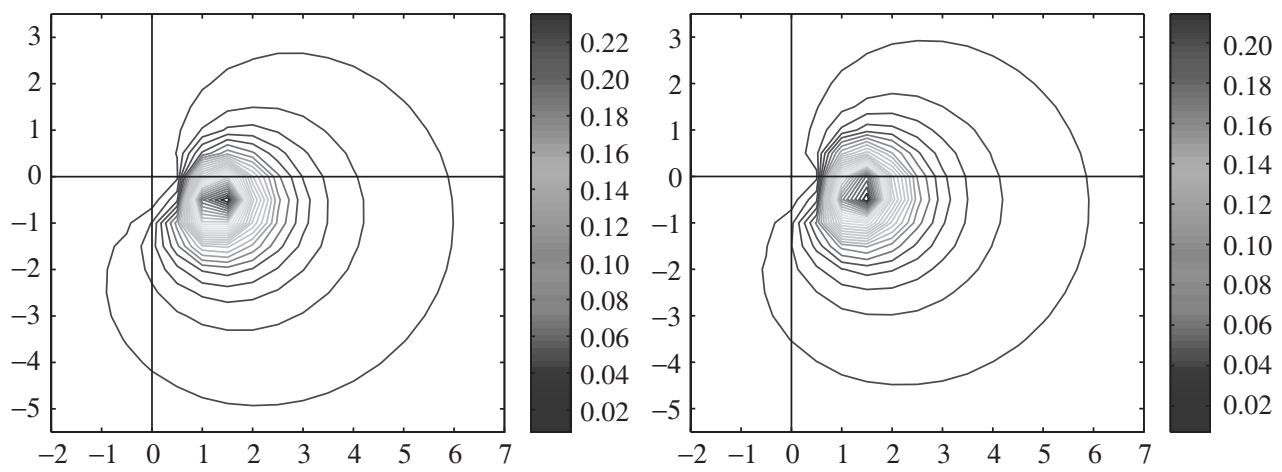

FIGURE 6: Level curves for the velocities in (left) spectrum 1 and (right) spectrum 2.

\section{Acknowledgement}

This research was partially financed by FONACIT, Proyecto Modelaje Estocástico Aplicado de la Agenda Petróleo.

\section{References}

AdLER, R. J. (1981). The Geometry of Random Fields. John Wiley, Chichester.

Azaïs, J.-M. AND Wschebor, M. (2005). On the distribution of the maximum of a Gaussian field with $d$ parameters. Ann. Appl. Prob. 15, 254-278.

Baxevani, A., Podgórski, K. And Rychlik, I. (2003). Velocities for random surfaces. Prob. Eng. Mechanics 18, 251-271.

Benzaquen, S. and Cabaña, E. M. (1982). The expected measure of the level set of a regular stationary Gaussian process. Pacific J. Math. 103, 9-16.

Berzin, C. And Wschebor, M. (1993). Approximation du temps local des surfaces gaussiennes. Prob. Theory Relat. Fields 96, 1-32.

Cabaña, E. M. (1985). Esperanzas de integrales sobre conjuntos de nivel aleatorios. In Proc. 2nd Latin American Congr. Prob. Math. Statist. (Caracas, 1985), Bernoulli Society, Caracas, pp. 65-82.

Cabaña, E. M. (1987). Affine processes: a test of isotropy based on level sets. SIAM J. Appl. Math. 47, 886-891.

Cramér, H. and Leadbetter, M. R. (1967). Stationary and Related Stochastic Processes. John Wiley, Chichester.

Corrsin, S. (1955). A measure of the area of a homogeneous random surface in space. Quart. Appl. Math. 12, $404-408$.

Federer, H. (1969). Geometric Measure Theory. Springer, New York.

Kratz, M. AND León, J. R. (2001). Central limit theorems for non-smooth functionals related to crossings (via the Hermite polynomial expansion). J. Theoret. Prob. 14, 639-671.

Krée, P. And Solze, C. (1983). Mécanique Aléatoire. Dunod, Paris.

Longuet-Higgins, M. S. (1957). The statistical analysis of a random moving surface. Phil. Trans. R. Soc. London A 249, 321-387.

OchI, M. (1998). Ocean Waves. Cambridge University Press.

Podgórski, K., Rychlik, I. AND SJÖ, E. (2000). Statistics for velocities of Gaussian waves. Internat. J. Offshore Polar Eng. 10, 91-98.

WsCHEBor, M. (1982). Formule de Rice en dimension $d$. Z. Wahrscheinlichkeitsth. 60, 393-401.

Wschebor, M. (1985). Surfaces Aléatoires (Lecture Notes Math. 1147). Springer, New York.

YlVISAKER, D. (1968). A note on the absence of tangencies in Gaussian sample paths. Ann. Math. Statist. 39, 261-262.

ZäHLE, U. (1984). A general Rice formula, Palm measures and horizontal-window conditioning for random fields. Stoch. Process Appl. 17, 265-283. 